\title{
Spin label scanning reveals likely locations of $\beta$-strands in the amyloid fibrils of the Ure2 prion domain
}

Jingzhou Wang, Giovanna Park, Yoon Kyung Lee, Matthew Nguyen, Tiffany San Fung, Tiffany Yuwen Lin, Frederick Hsu, and Zhefeng Guo*

Department of Neurology, Brain Research Institute, Molecular Biology Institute, University of California, Los Angeles, CA 90095, USA.

*To whom correspondence should be addressed: Zhefeng Guo, Department of Neurology, University of California, Los Angeles, 710 Westwood Plaza, Los Angeles, CA 90095. Phone:

(310) 439-9843; E-mail: zhefeng@ucla.edu 


\section{Supplemental Table}

Table S1. Values and fitting errors of Heisenberg exchange frequency $(\omega)$, rotational correlation time $(\tau)$ and order parameter $(S)$ from spectral simulations of spin-labeled Ure $2 \mathrm{p}_{1-89}-\mathrm{M}$ fibrils.

\begin{tabular}{|c|c|c|c|c|}
\hline \multirow{2}{*}{ Residue } & \multicolumn{3}{|c|}{ Component 1} & \multirow{2}{*}{$\begin{array}{c}\text { Component } 2 \\
\tau(\mathrm{ns})\end{array}$} \\
\hline & $\omega(\mathrm{MHz})$ & $\tau(\mathrm{ns})$ & $S$ & \\
\hline 2 & $58.7 \pm 0.61$ & $5.2 \pm 0.1$ & $0.6 \pm 0.01$ & $1.06 \pm 0.03$ \\
\hline 3 & $85.7 \pm 0.8$ & $7.3 \pm 0.1$ & $0.5 \pm 0.01$ & $1.2 \pm 0.05$ \\
\hline 4 & $71.7 \pm 0.7$ & $6.9 \pm 0.1$ & $0.5 \pm 0.01$ & $1.1 \pm 0.04$ \\
\hline 5 & $45.8 \pm 0.6$ & $7.04 \pm 0.1$ & $0.4 \pm 0.02$ & $1.4 \pm 0.08$ \\
\hline 6 & $86.2 \pm 0.9$ & $8.7 \pm 0.1$ & $0.4 \pm 0.4$ & $1.5 \pm 0.06$ \\
\hline 7 & $65.8 \pm 0.8$ & $6.98 \pm 0.1$ & $0.5 \pm 0.02$ & $1.1 \pm 0.03$ \\
\hline 8 & $136.9 \pm 1.2$ & $7.8 \pm 0.1$ & $0.4 \pm 0.01$ & \\
\hline 9 & $175.4 \pm 1.1$ & $6.6 \pm 0.1$ & $0.5 \pm 0.01$ & \\
\hline 10 & $122.8 \pm 0.8$ & $7.8 \pm 0.1$ & $0.1 \pm 0.05$ & \\
\hline 11 & $156.2 \pm 0.95$ & $6.03 \pm 0.09$ & $0.3 \pm 0.01$ & \\
\hline 12 & $97.9 \pm 0.5$ & $5.8 \pm 0.07$ & $0.4 \pm 0.02$ & \\
\hline 13 & $60.5 \pm 0.4$ & $6.7 \pm 0.09$ & $0.4 \pm 0.02$ & \\
\hline 14 & $85.8 \pm 0.4$ & $7.1 \pm 0.06$ & $0.2 \pm 0.03$ & \\
\hline 15 & $118.9 \pm 0.7$ & $6.7 \pm 0.08$ & $0.4 \pm 0.02$ & \\
\hline 16 & $144.5 \pm 0.9$ & $4.99 \pm 0.08$ & $0.6 \pm 0.007$ & \\
\hline 17 & $153.4 \pm 0.9$ & $5.5 \pm 0.08$ & $0.6 \pm 0.004$ & \\
\hline 18 & $151.05 \pm 0.9$ & $4.9 \pm 0.07$ & $0.6 \pm 0.01$ & \\
\hline 19 & $172.7 \pm 1.1$ & $6.1 \pm 0.1$ & $0.5 \pm 0.007$ & \\
\hline 20 & $157.5 \pm 1.2$ & $6.3 \pm 0.1$ & $0.5 \pm 0.01$ & \\
\hline 21 & $183.6 \pm 1.02$ & $4.7 \pm 0.07$ & $0.6 \pm 0.004$ & \\
\hline 22 & $125.5 \pm 0.7$ & $3.5 \pm 0.06$ & $0.6 \pm 0.008$ & \\
\hline 23 & $119.9 \pm 0.7$ & $3.2 \pm 0.07$ & $0.6 \pm 0.007$ & \\
\hline 24 & $195.8 \pm 1.02$ & $4.3 \pm 0.06$ & $0.6 \pm 0.004$ & \\
\hline 25 & $196.7 \pm 1.8$ & $5.9 \pm 0.2$ & $0.6 \pm 0.008$ & \\
\hline 26 & $159.98 \pm 0.8$ & $4.7 \pm 0.06$ & $0.5 \pm 0.006$ & \\
\hline 27 & $213.03 \pm 1.8$ & $6.9 \pm 0.2$ & $0.6 \pm 0.005$ & \\
\hline 28 & $208.4 \pm 1.6$ & $7.9 \pm 0.2$ & $0.5 \pm 0.006$ & \\
\hline 29 & $176.9 \pm 1.01$ & $5.9 \pm 0.08$ & $0.4 \pm 0.009$ & \\
\hline 30 & $224.2 \pm 1.9$ & $8.7 \pm 0.2$ & $0.5 \pm 0.007$ & \\
\hline 31 & $155.96 \pm 0.9$ & $5.5 \pm 0.09$ & $0.5 \pm 0.007$ & \\
\hline 32 & $124.9 \pm 0.6$ & $6.9 \pm 0.08$ & $0.5 \pm 0.009$ & \\
\hline 33 & $196.8 \pm 1.4$ & $8.8 \pm 0.1$ & $0.2 \pm 0.02$ & \\
\hline 34 & $160.2 \pm 0.8$ & $8.8 \pm 0.1$ & 0.0 & \\
\hline 35 & $191.5 \pm 1.1$ & $6.8 \pm 0.1$ & $0.53 \pm 0.005$ & \\
\hline 36 & $124.98 \pm 0.8$ & $6.06 \pm 0.09$ & $0.3 \pm 0.02$ & \\
\hline 37 & $151.9 \pm 0.8$ & $6.1 \pm 0.08$ & $0.4 \pm 0.008$ & \\
\hline 38 & $158.99 \pm 0.9$ & $5.4 \pm 0.08$ & $0.5 \pm 0.007$ & \\
\hline 39 & $114.7 \pm 0.5$ & $7.1 \pm 0.07$ & $0.3 \pm 0.01$ & \\
\hline 40 & $159.06 \pm 0.9$ & $5.3 \pm 0.08$ & $0.5 \pm 0.007$ & \\
\hline 41 & $149.6 \pm 0.8$ & $6.8 \pm 0.1$ & $0.5 \pm 0.007$ & \\
\hline
\end{tabular}




\begin{tabular}{|c|c|c|c|c|}
\hline 42 & 43.30 .9 & $4.4 \pm 0.1$ & $0.4 \pm 0.02$ & \\
\hline 43 & $99.96 \pm 0.7$ & $5.9 \pm 0.07$ & $0.2 \pm 0.02$ & \\
\hline 44 & $135.7 \pm 0.6$ & $5.06 \pm 0.06$ & $0.5 \pm 0.006$ & \\
\hline 45 & $201.9 \pm 1.4$ & $4.97 \pm 0.1$ & $0.6 \pm 0.006$ & \\
\hline 46 & $159.1 \pm 0.9$ & $5.5 \pm 0.09$ & $0.6 \pm 0.006$ & \\
\hline 47 & $93.1 \pm 0.4$ & $4.8 \pm 0.07$ & $0.5 \pm 0.01$ & \\
\hline 48 & $165.3 \pm 0.8$ & $5.6 \pm 0.07$ & $0.5 \pm 0.007$ & \\
\hline 49 & $149.1 \pm 0.5$ & $4.7 \pm 0.06$ & $0.5 \pm 0.007$ & \\
\hline 50 & $144.9 \pm 1.4$ & $7.5 \pm 0.2$ & $0.5 \pm 0.01$ & \\
\hline 51 & $132.8 \pm 0.7$ & $4.9 \pm 0.07$ & $0.5 \pm 0.008$ & \\
\hline 52 & $134.6 \pm 0.7$ & $3.97 \pm 0.05$ & $0.4 \pm 0.001$ & \\
\hline 53 & $130.95 \pm 0.6$ & $6.2 \pm 0.07$ & $0.5 \pm 0.008$ & \\
\hline 54 & $146.9 \pm 0.9$ & $5.3 \pm 0.08$ & $0.5 \pm 0.008$ & \\
\hline 55 & $136.8 \pm 0.8$ & $4.6 \pm 0.07$ & $0.4 \pm 0.01$ & \\
\hline 56 & $149.5 \pm 0.8$ & $4.7 \pm 0.06$ & $0.5 \pm 0.008$ & \\
\hline 57 & $157.1 \pm 0.8$ & $5.3 \pm 0.07$ & $0.5 \pm 0.006$ & \\
\hline 58 & $140.9 \pm 0.8$ & $4.4 \pm 0.06$ & $0.4 \pm 0.01$ & \\
\hline 59 & $121.4 \pm 0.7$ & $4.2 \pm 0.06$ & $0.5 \pm 0.001$ & \\
\hline 60 & $143.8 \pm 0.8$ & $5.1 \pm 0.06$ & $0.4 \pm 0.009$ & \\
\hline 61 & $125.7 \pm 0.7$ & $3.8 \pm 0.06$ & $0.5 \pm 0.008$ & \\
\hline 62 & $148.9 \pm 3.5$ & $5.2 \pm 0.1$ & $0.4 \pm 0.08$ & \\
\hline 63 & $160.6 \pm 0.9$ & $5.4 \pm 0.08$ & $0.5 \pm 0.007$ & \\
\hline 64 & $131.6 \pm 0.8$ & $4.3 \pm 0.07$ & $0.5 \pm 0.01$ & \\
\hline 65 & $151.7 \pm 0.9$ & $7.7 \pm 0.1$ & $0.4 \pm 0.01$ & \\
\hline 66 & $137.9 \pm 1.9$ & $6.02 \pm 0.1$ & $0.5 \pm 0.01$ & $1.2 \pm 0.05$ \\
\hline 67 & $185.3 \pm 2.3$ & $9.9 \pm 0.3$ & $0.6 \pm 0.005$ & $1.6 \pm 0.03$ \\
\hline 68 & $151.4 \pm 2.3$ & $6.9 \pm 0.2$ & $0.5 \pm 0.008$ & $1.4 \pm 0.04$ \\
\hline 69 & $70.7 \pm 1.1$ & $2.9 \pm 0.07$ & $0.4 \pm 0.02$ & $0.7 \pm 0.05$ \\
\hline 70 & $145.2 \pm 2.03$ & $13.7 \pm 0.5$ & $0.04 \pm 0.01$ & $1.5 \pm 0.03$ \\
\hline 71 & $129.2 \pm 2.5$ & $6.4 \pm 0.1$ & $0.5 \pm 0.01$ & $1.7 \pm 0.05$ \\
\hline 72 & $151.9 \pm 1.9$ & $9.5 \pm 0.2$ & $0.1 \pm 0.04$ & $0.6 \pm 0.04$ \\
\hline 73 & $75.2 \pm 0.8$ & $6.9 \pm 0.1$ & $0.6 \pm 0.001$ & $1.5 \pm 0.03$ \\
\hline 74 & $149.5 \pm 2.2$ & $13.3 \pm 0.4$ & $0.2 \pm 0.03$ & $1.8 \pm 0.06$ \\
\hline 75 & $173.6 \pm 1.8$ & $13.0 \pm 0.4$ & $0.5 \pm 0.01$ & $0.9 \pm 0.06$ \\
\hline 76 & $140.3 \pm 2.1$ & $7.3 \pm 0.2$ & $0.6 \pm 0.01$ & $1.5 \pm 0.03$ \\
\hline 77 & $38.3 \pm 1.2$ & $5.3 \pm 0.3$ & $0.7 \pm 0.02$ & $1.6 \pm 0.02$ \\
\hline 78 & $127.3 \pm 2.3$ & $8.9 \pm 0.3$ & $0.5 \pm 0.02$ & $1.6 \pm 0.05$ \\
\hline 79 & $71.8 \pm 1.4$ & $7.9 \pm 0.2$ & $0.4 \pm 0.02$ & $1.5 \pm 0.04$ \\
\hline 80 & $123.9 \pm 2.04$ & $9.1 \pm 0.2$ & $0.5 \pm 0.01$ & $1.98 \pm 0.08$ \\
\hline
\end{tabular}

